\title{
Perigos e riscos na medicina laboratorial: identificação e avaliação
}

\author{
Hazards and risks in laboratory medicine: identification and evaluation
}

Valéria Aparecida Faria'; Maria Leide de Sena Badaró2; Evelyn Rodrigues; Ricardo Hodja ${ }^{4}$; Maria Elizabete Mendes ${ }^{5}$; Nairo Massakazu Sumita ${ }^{6}$

unitermos
Perigos
Gestão de riscos
Saúde ocupacional
Acidentes do trabalho
Saúde do trabalhador
Laboratório clínico
Medicina laboratorial

\section{resumo}

Os perigos no ambiente de trabalho estão relacionados com qualquer tipo de fonte potencialmente danosa, em termos de lesões, ferimentos ou danos para a saúde ou uma combinação desses fatores. Os riscos são consequências dos perigos existentes no laboratório. Os laboratórios clínicos apresentam múltiplos riscos ocupacionais aos trabalhadores, categorizados como riscos biológicos, físicos, químicos, ergonômicos e para ocorrência de acidentes. É importante o laboratório identificar os riscos, avaliar os impactos que podem afetar o negócio e estabelecer critérios de priorização para a tomada de decisões, implementando estratégias e ações preventivas, a fim de evitar a instalação de falhas ou danos potenciais. Este artigo propõe uma sistemática de identificação e avaliação dos perigos e riscos em saúde e segurança ocupacional no laboratório clínico e discute suas aplicações na prática operacional.

Medicina laboratorial

abstract

Workplace hazards are related to any potentially harmful source in terms of lesions, injuries and health damage or a combination of these factors. The risks are consequences of laboratory hazards. Clinical laboratories pose multiple occupational hazards, which are categorized as biological, physical, chemical, ergonomic and accident prone. It is important to identify risks, assess the impacts that may affect the enterprise and establish prioritization criteria for making decisions. Furthermore, it is essential to implement strategies and preventive actions in order to avoid flaws or potential damage. Not only does this article propose a systematic identification and assessment of hazards, health risks and occupational safety within clinical laboratories, but it also discusses their applications in operational practice. key words

Dangers

Risk management

Occupational health

Accidents at work

Worker health

Clinical laboratory

Laboratory medicine

\footnotetext{
1. Bióloga; gestora de Saúde e Segurança Ocupacional da Divisão de Laboratório Central do Hospital das Clínicas da Faculdade de Medicina da Universidade de São Paulo (DLC/HC-FMUSP). 2. Bióloga; gestora do Plano de Atendimento à Emergência da Divisão de Laboratório Central da DLC/HC-FMUSP.

3. Bióloga; gestora de Meio Ambiente da Divisão de Laboratório Central da DLC/HC-FMUSP.

4. Engenheiro; mestre em Engenharia de Produção; diretor da Stance Cestão e Treinamento.

5. Doutora em Medicina (Patologia); médica patologista clínica; chefe da Seção Técnica de Bioquímica de Sangue da DLC/HC-FMUSP (LIM-03 da Patologia Clínica); coordenadora do Núcleo de Qualidade e Sustentabilidade da DLC/HC-FMUSP.

6. Doutor em Medicina; professor da disciplina de Patologia Clínica da FMUSP; médico patologista clínico; diretor do Serviço de Bioquímica Clínica da DLC/HC-FMUSP (LIM-03 da Patologia Clínica); assessor médico em Bioquímica Clínica do Fleury Medicina e Saúde.
} 


\section{Introdução}

A incorporação das boas práticas de relacionamento com empregados, sociedade, governo, acionistas, fornecedores e concorrentes é o caminho para as organizações alcançarem o sucesso nos negócios ${ }^{(20)}$. Isso faz que as organizações tenham foco nos aspectos relacionados com a qualidade de serviços e/ou produtos e a proteção ao meio ambiente, nas relações sociais, na saúde e na segurança de seus trabalhadores ${ }^{(14)}$.

$\mathrm{Na}$ área da saúde, em especial na medicina laboratorial, essa preocupação se repete e fica evidenciada no cotidiano da gestão. Para obter êxito em um laboratório clínico, é necessário dirigi-lo e controlá-lo de maneira transparente, ética e disciplinada, cumprindo-se a legislação(23).

É importante identificar os riscos e avaliar os impactos que podem afetar o negócio, a fim de que ações possam ser empreendidas preventivamente, evitando-se a instalação de falhas ou danos potenciais. A norma NBR ISO 31000:2009 - Gestão de riscos, princípios e diretrizes, da Associação Brasileira de Normas Técnicas (ABNT), orienta e facilita essa identificação em sua avaliação e na priorização para a tomada de decisões, propiciando a implantação de estratégias para melhorar a gestão de riscos laboratoriais(4).

A liderança precisa saber antecipar-se aos perigos, planejando-se e preparando-se para que as eventuais ameaças sejam contidas e o negócio se fortaleça ${ }^{(25)}$. Assim, o laboratório torna-se capaz de identificar e proteger seus produtos e serviços críticos, ativando sua capacidade de gerir os incidentes, preparando seus colaboradores para atuarem corretamente nessas situações e protegendo a imagem da empresa ${ }^{(37)}$.

Perigo no ambiente de trabalho é definido como qualquer fonte, situação ou ato com potencial para dano em termos de lesões, ferimentos ou danos para a saúde ou uma combinação destes ${ }^{(3)}$. Já o risco é consequência do perigo. Portanto, se os perigos estiverem ausentes, não há riscos no ambiente de trabalho. Como isso é praticamente impossível, trabalha-se na minimização do risco e dos impactos por meio de ações de bloqueio, mecanismos de controle e educação dos envolvidos, buscando-se maiores níveis de conscientização para as questões de saúde e segurança ocupacionais.

O laboratório clínico é um lugar com inúmeras fontes de perigo. Por isso, torna-se relevante o levantamento dos perigos para identificá-los adequadamente e avaliar os riscos a que estão submetidos trabalhadores, terceiros que prestam serviços em suas instalações e vizinhança. Em consequência, os riscos no laboratório são multidimensionais, tanto do ponto de vista da estabilidade como da previsibilidade dos resultados. A gestão de riscos envolve necessariamente todos os níveis da empresa ${ }^{(37)}$.

O objeto desta discussão é alertar para a questão da sustentabilidade e da continuidade da realização dos serviços, que se relaciona com a implantação e a manutenção de um ambiente saudável no laboratório ${ }^{(34)}$.

A norma Occupational Health and Safety Assessment Series (OHSAS) 18001:2007(10) contribui para o planejamento no reconhecimento dos perigos, na identificação dos riscos de exposição e, essencialmente, na promoção de ações, orientando a introdução de mecanismos de controle operacionais, o monitoramento das ações de bloqueio e o estabelecimento de instruções de trabalho associadas ao assunto.

Considerar todos esses fatores de exposição dos trabaIhadores em um sistema de gestão de saúde e segurança ocupacional é fundamental para o desenvolvimento de boas práticas e vigilância na saúde do trabalhador, criando-se um ambiente de trabalho seguro( ${ }^{(5)}$.

Os perigos mais comuns no cotidiano podem ser divididos em físicos, químicos, biológicos, ergonômicos e acidentes. Durante a jornada de trabalho, os funcionários do laboratório estão continuamente expostos a situações de risco, que podem gerar danos à sua saúde.

No Brasil, existem inúmeros convênios e recomendações da Organização Internacional do Trabalho (OIT), ratificadas pelas Portarias do Ministério do Trabalho, denominadas Normas Regulamentadoras (NRs), além da Consolidação das Leis do Trabalho (CLT), disciplinando essa área. Os estudos sobre riscos ocupacionais afirmam que, quando eles não são controlados, geram acidentes e doenças profissionais e do trabalho ${ }^{(24)}$. O Ministério do Trabalho, por meio das NRs, visa eliminar ou controlar esses riscos ocupacionais.

Objetivamente, os agravos à saúde relacionados com o trabalho estão classificados em dois grupos. No primeiro, correlacionados com aspectos de segurança no ambiente de trabalho, incluem-se aqueles que demonstram a ruptura abrupta do equilíbrio entre as condições, o ambiente de trabalho e a saúde do trabalhador, como os acidentes do trabalho e as intoxicações agudas de origem profissional(26). O segundo grupo inclui os agravos de caráter crônico, isto é, a doença profissional típica, definida como inerente ou peculiar a determinado ramo de atividade ${ }^{(26)}$. Essa categoria envolve as doenças relacionadas com o trabalho, que a 
Organização Mundial da Saúde (OMS) define como "agravos outros que, em adição a doenças profissionais legalmente desconhecidas, ocorrem em trabalhadores quando o ambiente ou condições contribuem significativamente para a ocorrência de doenças, porém em graus variados de magnitude"(33).

Os riscos físicos no laboratório envolvem a exposição excessiva a ruídos (p. ex., centrifugação de materiais biológicos e automação laboratorial), radiações ionizantes (por meio da manipulação de radioimunensaios $)^{(8,9)}$ e variações de temperatura (ar condicionado, câmaras frias, autoclaves).

Profissionais de saúde têm mais riscos de exposição a certas doenças infecciosas transmissíveis por via respiratória, devido à exposição a sangue e fluidos orgânicos, por via fecal-oral e por contato(16). A exposição ocupacional ao material biológico representa um risco aos trabalhadores dos laboratórios clínicos, devido à possibilidade de transmissão de patógenos, como os vírus das hepatites $B$ e C (HBV e HCV) e o vírus da imunodeficiência humana $(\mathrm{HIV})^{(12,21,24,29,38)}$. As consequências dessa exposição podem afetar diretamente os trabalhadores, atingindo-os nos aspectos físico, psicológico, familiar e social(22).

Nos EUA, estima-se que existam cerca de 8 milhões de trabalhadores da saúde vítimas de acidentes com material perfurocortante. No período de 1995 a 2001, foram registrados 16.922 acidentes dessa natureza distribuídos da seguinte forma: enfermeiras $44 \%$, médicos $28 \%$, técnicos de laboratório $15 \%$, estudantes $4 \%$ e pessoal de limpeza $3 \%{ }^{(13)}$. No Brasil, em razão da carência de estatísticas consolidadas e oficiais sobre o assunto, foi criada a Rede de Prevenção de Acidentes do Trabalho com Material Biológico em Hospitais Brasileiros (REPAT), cujas metas são o controle e a prevenção de acidentes do trabalho com exposição a material biológico $(35,36)$.

As avaliações de risco e vigilância em saúde para a exposição a agentes químicos no ambiente de trabalho se justificam pelo crescimento do uso de substâncias químicas nas atividades de produção, armazenamento e transporte ${ }^{(15,42)}$. Isso também ocasionou um impacto marcante no meio ambiente e na saúde do homem, tanto em razão da exposição ocupacional quanto da contaminação ambiental decorrente deles ${ }^{(17)}$. Em nível global, isso provocou aumento no número de trabalhadores expostos a esses riscos. Porém, nem sempre a exposição a produtos químicos resulta em efeitos prejudiciais à saúde. Ela dependerá de fatores como tipo de agente químico e sua concentração, frequência e duração da exposição, práticas e hábitos laborais e suscetibilidade individual(15).
Acidentes químicos correspondem a eventos agudos, como explosões, incêndios e emissões, atuando individualmente ou combinados, envolvendo uma ou mais substâncias perigosas, com potencial de causar simultaneamente múltiplos danos ao meio ambiente e à saúde dos seres humanos expostos. A ampliação das consequências desses acidentes não se restringe somente à possibilidade de causar óbito, mas engloba o potencial da gravidade e a extensão de seus efeitos, que podem ultrapassar os limites espaciais (laboratório, vizinhança, cidade) e temporais (teratogênese, carcinogênese, mutagênese e danos a órgãos-alvo específicos) ${ }^{(6,7,15,17,41)}$.

No laboratório, há outros fatores de exposição, como os ergonômicos, mecânicos e de acidentes, que também impactam a integridade da saúde dos trabalhadores.

Os riscos ergonômicos e psicossociais no laboratório decorrem da organização e da gestão do trabalho; podem ser apontados os esforços repetitivos, os turnos diferenciados de trabalho e o controle rígido da produtividade ${ }^{(24)}$. As medidas ergonômicas relacionadas com a postura no ambiente de trabalho, assim como as soluções implementadas preventivamente, são mais positivas, especialmente quando associadas à utilização de técnicas corretas no processo de trabalho ${ }^{(28,39)}$.

Este artigo visa apresentar uma sistemática para a identificação dos perigos e uma avaliação dos riscos à saúde e à segurança ocupacional, com foco nas atividades desenvolvidas no laboratório clínico. O papel da norma OHSAS 18001:2007 e a repercussão de sua implantação na gestão de processos no laboratório clínico também serão criticamente avaliados.

\section{Identificação dos perigos}

Para cada macroprocesso, é preciso conhecer atividades e situações presentes em suas operações, regime da atividade, perigos relacionados, danos potenciais, controles vigentes (equipamentos de proteção coletiva e individual, procedimentos, sinalizações) e postura em relação à segurança. A identificação de perigos considera as interações dos processos com funcionários, contratados, visitantes, clientes e vizinhança. Nela, o comportamento humano precisa ser considerado, devido à possibilidade de adesão ou não aos programas de Saúde e Segurança Ocupacional (SSO).

Os perigos devem ser avaliados em todas as situações, corriqueiras/normais do cotidiano laboratorial ou anormais, e para os potenciais acidentes que podem vir a acontecer 
no âmbito laboratorial. Deve-se observar, nessa avaliação, a existência ou não de legislação específica aplicável ao ambiente de trabalho que está tendo seus perigos e riscos avaliados. É importante considerar o layout do local de trabalho, os processos, as instalações, os equipamentos, os procedimentos operacionais e a organização do trabalho, o qual deve ser revisto periodicamente, condicionando-se a remodelações nos processos, inovações de área física e/ou introdução de novas atividades. A responsabilidade pelo levantamento é dos gestores em conjunto com os responsáveis de cada atividade.

O detalhamento dos danos potenciais é importante porque são essas as informações que auxiliarão a formação de critérios a serem usados na classificação dos riscos. Para facilitar essa tarefa, recomenda-se a preparação prévia de uma tabela com os perigos e danos correspondentes. Nela descrevem-se a natureza, o tipo do perigo e o dano correspondente com a pontuação para seu nível de severidade - discretamente prejudicial (1), prejudicial (2) e extremamente prejudicial (3).

\section{Cálculo do risco por meio do estabelecimento de pontuações}

Frequência das atividades desenvolvidas: pode ser desde ocasional (menos de 10 vezes por ano) a muito frequente (uma ocorrência ao dia).

Duração: varia de curta (inferior a uma hora) a longa duração (superior a 8 horas).

Probabilidade de o dano vir a ocorrer: improvável $=1$ ponto; pouco provável = 2 pontos; provável $=3$ pontos.

Avaliação do comportamento humano: altamente compromissado $=1$ ponto; compromissado $=2$ pontos; requer treinamento $=3$ pontos.

Cálculo do risco: o resultado final é obtido multiplicando-se a pontuação de cada item: severidade do dano $x$ ocorrência da atividade $x$ probabilidade de o dano vir a ocorrer $\times$ comportamento humano.

\section{Resultado da avaliação}

O valor aceitável é variável e cada laboratório deverá defini-lo de acordo com suas características. Para os itens que suplantarem os limites de aceitação deverão, necessariamente, ser abertos planos de ações corretivas ou preventivas, com a finalidade de minimizar esses riscos. Classificam-se os resultados obtidos em risco: não aceitável, aceitável com atenção e aceitável.

\section{Exemplos práticos}

Atividade: centrifugação de material biológico na área de triagem de materiais de um laboratório clínico.

\section{Tabela 1 Perigos e danos}

\begin{tabular}{|c|c|}
\hline Natureza do perigo & Exposicão a materiais biológicos \\
\hline Tipo de perigo & $\begin{array}{l}\text { Exposição a vírus, fungos, } \\
\text { bactérias, parasitas e } \\
\text { protozoários }\end{array}$ \\
\hline Dano & Doença infectocontagiosa \\
\hline Severidade do dano & Prejudicial (pontuação = 2) \\
\hline
\end{tabular}

\section{Tabela 2 Critérios definidos pelo laboratório}

\begin{tabular}{|c|c|}
\hline Não aceitável & Acima de 50 pontos \\
\hline $\begin{array}{l}\text { Aceitável com nível } \\
\text { de atenção }\end{array}$ & Entre 40 e 50 pontos \\
\hline Aceitação & Abaixo de 40 pontos \\
\hline Severidade do dano & Prejudicial (pontuação = 2) \\
\hline
\end{tabular}

\section{Tabela 3 Cálculo de risco}

\begin{tabular}{lll}
\hline Severidade do dano & Prejudicial (2) \\
\hline $\begin{array}{l}\text { Frequência } \\
\text { Duração }\end{array}$ & Muito frequente (3) \\
\hline $\begin{array}{l}\text { Probabilidade de o } \\
\text { dano vir a ocorrer }\end{array}$ & Provável (3) \\
$\begin{array}{l}\text { Comportamento } \\
\text { humano }\end{array}$ & Compromissado (2) \\
$\begin{array}{l}\text { Resultado final } \\
\text { Análise crítica }\end{array}$ & $\begin{array}{l}2 \times 3 \times 1 \times 3 \times 2=36 \text { pontos } \\
\text { Resultado aceitável, portanto } \\
\text { não requer abertura de planos de } \\
\text { ação }\end{array}$ \\
\hline
\end{tabular}

\section{Aplicando o ciclo do PDCA (plan, do, check, action)}

Após a avaliação, há a abertura de planos de ação para conceber, manter ou melhorar os controles da atividade. A gestão de mudanças ${ }^{(18)}$ propicia que o ciclo do PDCA ${ }^{(40)}$ seja aplicado para que os riscos diminuam. Ela permite uma abordagem equilibrada dos aspectos técnicos e organizacionais, visando reduzir as possíveis resistências e obter uma transformação mais eficaz, completa e em menor tempo.

Os riscos podem ser reduzidos a partir da seguinte hierarquia: eliminação, substituição, controles de engenharia, sinalizações de riscos e equipamento de proteção individual. 
As mudanças impactam vários aspectos no laboratório, como cultura, métodos de trabalho, tecnologias e formas como os indivíduos interagem entre si, e os processos sobre os quais passarão a atuar.

\section{Discussão}

No Brasil, como já ocorre em diferentes países, a tendência é que a legislação crie um arcabouço jurídico ${ }^{(19)}$, em que as empresas sejam obrigadas a demonstrar à fiscalização, de maneira sistemática e proativa, os perigos identificados em seu processo, e não apenas nos esforços ocasionais para controlar os riscos ${ }^{(1)}$. Os serviços de saúde devem atender, obrigatoriamente, às NRs número 9 (Programa de Prevenção de Riscos Ambientais) e número 32 (Segurança e Saúde no Trabalho de Serviços de Saúde) do Ministério do Trabalho e Emprego (MTE) $)^{(30-32)}$.

Em uma parcela significativa dos laboratórios clínicos brasileiros, o tratamento para questões relacionadas com segurança, medicina do trabalho e saúde ocupacional restringe-se à coleta de dados estatísticos, ações reativas a acidentes do trabalho e respostas a causas trabalhistas, o que denota que ainda há uma visão restrita em relação ao assunto ${ }^{(34)}$.

O histórico estatístico do Instituto Nacional de Previdência Social (INSS) é alarmante. Em 2009, foram registrados 723.452 acidentes e doenças do trabalho entre os trabalhadores assegurados, excluindo-se os profissionais autônomos e os domésticos. Esses eventos provocam grande impacto social, econômico e sobre a saúde pública no Brasil. Foram contabilizadas 17.693 doenças relacionadas com o trabalho. Parte desses acidentes e doenças tiveram como consequência o afastamento das atividades de 623.026 indivíduos. Desse total, o número de trabalhadores com afastamento temporário de até 15 dias, devido à incapacidade, corresponde a 302.648; com tempo de afastamento superior a 15 dias, 320.378; por incapacidade permanente, 13.047; e óbito de 2.496 cidadãos $^{(27)}$.

Os indicadores de quantidade de acidentes de trabalho por situação do registro e motivo estão listados em um total de 47 subgrupos; o maior índice pertence à categoria dos profissionais de serviços gerais (76.256), ou seja, 10,5\% do total. Os profissionais da saúde totalizam 35.462 (4,9\%) registros de acidente de trabalho, sendo que os técnicos de nível médio da saúde representam 4,1\% (29.718) e os profissionais de nível superior em ciências biológicas, 0,79\% do total (5.744 ocorrências) ${ }^{(27)}$.
O custo operacional do INSS somado às despesas na área da saúde e afins atinge um valor na ordem de $R \$ 57$ bilhões. A dimensão dessa cifra apresenta a premência na adoção de políticas públicas voltadas à prevenção e à proteção contra os riscos relativos às atividades laborais ${ }^{(27)}$.

Diante desse cenário, o governo está direcionando a fiscalização para setores econômicos com maior taxa de frequência de acidentes, ampliando a participação da sociedade produtiva nas propostas de modernização da legislação trabalhista, com o objetivo de reduzir as situações de risco(2).

Com base no exposto, o investimento em um sistema de gestão de segurança e saúde ocupacional pode ser considerado uma ação favorável ao encontro da sustentabilidade e do exercício da responsabilidade social, cujo objetivo é eliminar e reduzir os impactos dos acidentes sobre os trabaIhadores, suas famílias, o governo e a sociedade no geral ${ }^{(1)}$.

Com o conhecimento desses fatores de riscos e a identificação da população exposta (os próprios profissionais, prestadores de serviços e clientes), cabe aos gestores lutar pela orientação do trabalhador sobre esses riscos ocupacionais e as medidas necessárias a seu controle. A atuação das Comissões Internas para Prevenção de Acidentes (CIPAs), em conjunto com o Serviço Especializado em Engenharia de Segurança e Medicina do Trabalho (SESMT), facilita a discussão dessa problemática com a direção dos serviços laboratoriais.

Um programa de educação continuada, com treinamento específico para os profissionais de laboratório, ajuda no entendimento desses problemas e de sua gravidade para combatê-los.

\section{Conclusão}

Há medidas específicas de proteção à saúde do trabaIhador que são quantitativa e qualitativamente satisfatórias e devem ser implantadas. É uma questão de cidadania a conquista desse direito do trabalhador.

Foi apresentado um instrumento de identificação de perigos e avaliação dos riscos no laboratório, que demonstrou ser prático e fácil de ser aplicado pelos envolvidos.

A equipe do laboratório clínico precisa ser mobilizada para aplicar as medidas em favor de sua própria saúde, da produtividade do serviço, de seu melhor desempenho e da satisfação no trabalho.

Destaca-se como elemento de grande eficiência o domínio do conhecimento de fatores de riscos ocupacionais, causas das patologias do trabalho e medidas de controle. 


\section{Referências}

1. ALVES, D. B. Condições de trabalho na enfermagem: aspectos teóricos. In: CONGRESSO BRASILEIRO DE ENFERMGEM. Anais. Salvador, p. 39, 1988.

2. ARAÚJO, G. M. Normas regulamentadoras comentadas: legislação de segurança e saúde no trabalho. Rio de Janeiro: GVC, 2007.

3. ASSOCIAÇÃO BRASILEIRA DE NORMAS TÉCNICAS. NBR 18801. Sistema de gestão da segurança e saúde no trabalho: requisitos. Rio de Janeiro, 2011.

4. ASSOCIAÇÃO BRASILEIRA DE NORMAS TÉCNICAS. NBR ISO 31000. Gestão de riscos, princípios e diretrizes. Rio de Janeiro, 2009.

5. BENITE, A. G. Sistema de gestão da segurança e saúde do trabalho em empresas da construção. 2004. Dissertação (Mestrado Politécnico) - USP, São Paulo, 2004.

6. BERTAZZI, P. A. et al. Cancer incidence in a population accidentally exposed to 2,3,7,8-tetrachlorodibenzo-pdioxin. Epidemiology, v. 4, p. 398-406, 1993.

7. BINDER, M. C. P.; ALMEIDA, I. M.; MONTEAU, M. Árvore de causas. Método de investigação e análise de acidentes de trabalho. São Paulo: Publisher Brasil Editora, 2000. $144 \mathrm{p}$.

8. BRENNER, D. J. et al. Cancer risks attributable to low doses of ionizing radiation: assessing what we really know. Proc Natl Acad Sci USA, v. 100, n. 24, p. 13761-6, 2003.

9. BRENNER, D. J.; SACHS, R. K. Estimating radiation-induced cancer risks at very low doses: rationale for using a linear no-threshold approach. Radiat Environ Biophys, v. 44, n. 4, p. 253-6, 2006.

10. BS OHSAS 18001. Occupational Health and Safety Assessment Series. BSI, 2007.

11. CAIXETA, R. B.; BARBOSA-BRANCO, A. Acidente de trabalho, com material biológico, em profissionais de saúde de hospitas públicos do Distrito Federal, Brasil, 2002/2003. Caderno de Saúde Pública, v. 21, n. 3, p. 737-46, 2005.

12. CANINI, S. R. M. S. et al. Acidentes perfurocortantes entre trabalhadores de enfermagem de um hospital universitário do interior paulista. Rev Latino-Am Enfermagem, v. 10, n. 2, p. 172-8, 2002.

13. CENTERS FOR DISEASE CONTROL AND PREVENTION (CDC). Guideline for infection control in health care personnel. Infect Control Hosp Epidemiol, v. 19, n. 6, p. 455, 2001.

14. CHAIB, E. B. Proposta para implementação de sistema de gestão integrada de meio ambiente, saúde e segurança do trabalho em empresas de pequeno e médio porte: um estudo de caso da indústria metal-mecânica. 2005. Dissertação (Mestrado; Programa de Pós-graduação em Engenharia) - Universidade Federal do Rio de Janeiro, Rio de Janeiro, 2005.

15. COSTA, K. N. S. et al. Avaliação dos riscos associados ao uso do xilol em laboratórios de anatomia patológica e citologia. Rev Bras Saúde Ocup, São Paulo, v. 32, n. 116, p. 50-6, 2007.

16. Departamento de Vigilância Epidemiológica. Secretaria de Vigilância em Saúde. Ministério da Saúde. Manual dos centros de referência para imunobiológicos especiais. Brasília, DF: Ministério da Saúde; Departamento de Vigilância Epidemiológica, 2006. 188 p. (Série A. Normas e Manuais Técnicos).

17. FREITAS, C. M.; PORTO, M. F. S.; MOREIRA, J. C. Segurança química, saúde e ambiente: perspectivas para a governança no contexto brasileiro. Cad Saúde Pública, v. 18, n. 1, p. 249-56, 2002.

18. GROL, R.; GRIMSHAW, J. From best evidence to best practice: effective implementation of change in patients' care. Lancet, v. 362, p. 1225-30, 2003.

19. GUNNINGHAM, N.; JOHNSTONE, R. Regulating workplace safety: sistem and sections. New York: Oxford University Press Inc., 1999.

20. MACHADO, J. Processo de vigilância em saúde do trabalhador. Caderno de Saúde Pública, v. 13, Supl. 2, p. 33-45, 1997.

21. MARZIALE, M. H. P. et al. Acidentes com material biológico em hospital da Rede de Prevenção de Acidentes do Trabalho (REPAT). Revista Brasileira de Saúde Ocupacional, v. 32 n. 115, p. 109-19, 2007.

22. MARZIALE, M. H. P.; NISHIMURA, K. Y. N.; FERREIRA, M. M. Riscos de contaminação ocasionados por acidentes de trabalho com material pérfuro-cortante entre trabalhadores de enfermagem. Rev Latino-Am Enfermagem, v. 12, n. 1, p. 36-42, 2004.

23. MASSOCO, D. B. Uso da metodologia árvore de causas na investigação de acidente rural. Dissertação (Mestrado) - Universidade Federal de Santa Maria, Santa Maria, RS, 2008.

24. MAURO, M. Y. C. et al. Riscos ocupacionais em saúde. $R$ Enferm UERJ, v. 12, p. 338-45, 2004.

25. MENDES, M. E. et al. Gestão por processos no laboratório clínico: uma abordagem prática. São Paulo: EPR Editora Ltda., 2006. cap. 1. p. 13-36.

26. MENDES, R. (Org.). Patologia do trabalho. Rio de Janeiro: Atheneu, 1995.

27. MINISTÉRIO DA PREVIDÊNCIA SOCIAL. Anuário estatístico da Previdência Social. Disponível em: <http:// www. previdenciasocial.gov.br/conteudoDinamico. php?id=423>. Acesso em: 5 maio 2011.

28. MINISTÉRIO DA SAÚDE. Organização Pan-Americana da Saúde no Brasil. Doenças relacionadas ao trabalho: manual de procedimentos para os serviços de saúde. Brasília, DF: OPAS/OMS, 2001.

29. MINISTÉRIO DA SAÚDE. Secretaria de Políticas de Saúde. Coordenação Nacional de DST e AIDS. Manual de condutas: exposição ocupacional a material biológico: hepatite e HIV. Brasília, DF, 1999.

30. MINISTÉRIO DO TRABALHO E EMPREGO. Norma Regulamentadora $n^{\circ}$ 7. Programa de controle médico de saúde ocupacional, de 29 de dezembro de 1994.

31. MINISTÉRIO DO TRABALHO E EMPREGO. Norma Regulamentadora no 9. Programa de prevenção de riscos ambientais, de junho de 1978 (revisão 1990).

32. MINISTÉRIO DO TRABALHO E EMPREGO. Norma Regulamentadora $n^{\circ}$ 32. Segurança e saúde no trabalho em serviços de saúde, de 29 de dezembro de 1994. 
33. OLIVEIRA, B. R.; MUROFUSE, N. T. Acidentes de trabalho e doença ocupacional: estudo sobre o conhecimento do trabalhador hospitalar dos riscos à saúde de seu trabalho. Rev Latino-Am Enfermagem, Ribeirão Preto, v. 9, n. 1, p. 109-15, 2001.

34. QUELHAS, O.; LIMA, G. Sistema de gestão de segurança e saúde ocupacional: fator crítico de sucesso à implantação dos princípios do desenvolvimento sustentável nas organizações brasileiras. INTERFACEHS - Revista de Gestão Integrada em Saúde do Trabalho e Meio Ambiente, v. 1, n. 2, artigo 2.

35. REDE DE PREVENÇÃO DE ACIDENTE DE TRABALHO (REPAT). Universidade de São Paulo. Escola de Enfermagem de Ribeirão Preto. Disponível em: <http:// www.eerp.usp.br/repat>. Acesso em: 11 maio 2011.

36. SÃO PAULO. Secretaria de Estado da Saúde. Programa DST/AIDS-SP. Vigilância de acidentes com material biológico. Bol Epidemiol SINABIO, p. 20, 2002.

37. SBPC/ML. Gestão de riscos no laboratório clínico. In: Gestão da fase pré-analítica: recomendações da
Sociedade Brasileira de Patologia Clínica/Medicina Laboratorial (SBPC/ML). Rio de Janeiro, 2010. p. 1-36.

38. SHAPIRO, C. N. Occupational risk of infection with hepatitis $B$ and hepatitis C virus. Surg Clin North Am, v. 6, n. 75, p. 1047-56, 1995.

39. SILVA, E. F. Estudo sobre acidentes de trabalho ocorridos com trabalhadores de enfermagem de um hospital de ensino. Dissertação (Mestrado) - Universidade de São Paulo, São Paulo, 1998.

40. SILVA, L. C. et al. Gestão ambiental e tecnologias ambientais: práticas e benefícios em uma indústria alimentícia no sul da Bahia. RGSA, v. 4, n. 1, p. 80-91, 2010.

41. WEYNE, G. R. S. Lições dos grandes desastres das indústrias químicas de Flixborough, Seveso e Bophal. Saúde e Trabalho, v. 2, p. 3-13, 1988.

42. XELEGATI, R.; ROBAZZI, M. Riscos químicos a que estão submetidos os trabalhodores de enfermagem: uma revisão de literatura. Revista Latino-Am Enfermagem, v. 11, n. 3, p. 350-6, 2003. 